\title{
Observer-Based Robust Control of Uncertain Switched Fuzzy Systems with Combined Switching Controller
}

\author{
Hong Yang, ${ }^{1}$ Huanhuan Lü,, ${ }^{1}$ and Xiaodong Liu' ${ }^{2}$ \\ ${ }^{1}$ School of Information Engineering, Shenyang University, Shenyang 110044, China \\ ${ }^{2}$ School of Electronic and Information Engineering, Dalian University of Technology, Dalian 116023, China \\ Correspondence should be addressed to Hong Yang; cherryyh@126.com
}

Received 1 April 2013; Accepted 27 May 2013

Academic Editor: Xiaojie Su

Copyright (c) 2013 Hong Yang et al. This is an open access article distributed under the Creative Commons Attribution License, which permits unrestricted use, distribution, and reproduction in any medium, provided the original work is properly cited.

\begin{abstract}
The observer-based robust control for a class of switched fuzzy (SF) time-delay systems involving uncertainties and external disturbances is investigated in this paper. A switched fuzzy system, which differs from existing ones, is firstly employed to describe a nonlinear system. Next, a combined switching controller is proposed. The designed controller based on the observer instead of the state information integrates the advantages of both the switching controllers and the supplementary controllers but eliminates their disadvantages. The proposed controller provides good performance during the transient period, and the chattering effect is removed when the system state approaches the origin. Sufficient condition for the solvability of the robust control problem is given for the case that the state of system is not available. Since convex combination techniques are used to derive the delay-independent criteria, some subsystems are allowed to be unstable. Finally, various comparisons of the elaborated examples are conducted to demonstrate the effectiveness of the proposed control design approach.
\end{abstract}

\section{Introduction}

In practical control systems, the nonlinear property, uncertainty, and time delay exist widely, because of the complexity of control procedure, disturbances, and the constraint of system. Therefore, the stabilization and control issues for nonlinear time-delay systems with uncertainties are very important issues, and they are challenging and important in both theory and practical applications [1-5].

Also recently, the switched systems, which are an important class of hybrid systems and have wide background and technological applications, have also been one of the main research focuses. In turn, a considerable number of fruitful results in analysis and design of switching systems have been derived too; for example, see [6-13]. On the other hand, the research activities on the synergy of fuzzysystem-based controls, as an important intelligent control approach, combined with some of math-analytical control theories have attracted great attention. In particular, the class of Takagi-Sugeno (T-S) fuzzy models has been found to be most effective as system model in various fuzzy-system-based methods. Based on T-S fuzzy model representations and the feedback control strategy, stability and robust analysis and design as well as handling parameter uncertainties for fuzzy systems have acquired a considerable number of fruitful results; for example, see [14-26].

Recently, the stabilizability conditions and smoothness conditions for fuzzy switching control systems were reported. Based on the T-S fuzzy systems, Tanaka et al. [16-19] introduced new switching fuzzy systems for more complicated real systems such as multiple nonlinear systems, switched nonlinear hybrid systems, and second-order nonholonomic systems. This class of the model has two levels of structures. It will switch between the second-level region rules according to the first-level region rules. In fact, it is a type of switching for the same premise variable.

To effectively achieve nonlinear control, we propose an SF model in this paper [27]. Here, by assumption, TakagiSugeno fuzzy systems, which are also denoted as T-S fuzzy systems, are considered as terms of plant representation models. These fuzzy systems belong to the category of datadriven fuzzy systems, in contrast to the more traditional 
category of semantic-driven fuzzy models. The proposed corresponding model of SF systems, presented below, differs from the existing ones cited in the literature in the fact that each subsystem is a T-S fuzzy system, hence defining a class of SF systems. It should be noted that this class inherits some essential features of hybrid systems while retaining all of the information and knowledge representation capacity of fuzzy systems. Furthermore, this model is used for the extra design of the switching law based on the previous works [16-19].

Comparing with the previous works [16-19], this model of SF systems does not have two levels of structures, but it is switching between each of the sub fuzzy systems, not depending on region fuzzy rules. In this case, stability analysis is often facilitated by the fact that properties of each individual sub fuzzy system are of concern only in the regions where this subsystem is active, and the behavior of the sub fuzzy systems in other parts of the state space has no influence on the SF system. It is possible to find a switching law that renders the SF systems stable for unstable sub fuzzy systems. Particularly, when the parameters suddenly change or discontinuously change, the switching rules can be designed as any combinational function of variables, which make up the insufficiency of the switching depending on the single variable of the switching fuzzy model [16-19].

Control of SF systems is a challenging task because no systematic mathematical tools exist to help find necessary and sufficient conditions to guarantee the stability and performance. The problem becomes more complex if some of the system's parameters are uncertain, especially observer-based control. The study of observer-based control, which has been well addressed for switched systems with delay [28-30] and fuzzy systems with delay [31-33], has been rarely investigated for SF systems. In [34], although the observer-based control of SF systems has been investigated, the external disturbance is not considered. However, to our knowledge, there are no papers considering observer-based control of SF timedelay systems with the external disturbance. Observer-based control for the SF system which is based on designed switching method is still an open and interesting issue.

It is well known that parallel distributed compensation (PDC) control has been found to be a particular means for fuzzy systems due to its universal property [16-19, 34]. However, an undesired chattering effect will occur. This paper presents a combined switching controller, which has nonfuzzy-rules-based combination of switching controller and supplementary controllers, for SF systems subject to parameter uncertainties. The switching controller consists of several linear controllers. One of the switching controllers is employed at each moment according to a switching scheme, which is derived based on the Lyapunov stability theory. The supplementary controller is employed to handle the external disturbance. The smooth transition acted on the switching controller and the supplementary controllers is governed. As a result, the combined switching controller provides good performance during the transient period, and the chattering effect is removed when the system state approaches the origin.

In this paper, we investigate the problem of observerbased robust control for SF systems involving uncertainties and external disturbances with time delay. Sufficient condition for the solvability of the robust control problem is given for the case that the state of a system is unmeasurable. The design of a switching control law based on observer state instead of the original state information is considered. We use the multiple Lyapunov function technique to design a combined switching controller and a switching law such that the observer performance is satisfied. The feasibility of the problem can be realized by convex combination techniques and linear matrix inequalities. Finally, the simulation examples show the validity of the proposed design method.

This paper is organized as follows. Section 2 introduces a switching fuzzy system and an SF system. In Section 3, we describe the model of an SF system of time-delay case involving uncertainties and external disturbances. In Section 4, state observer and the combined switching controller are derived. Two compared example simulations will be presented in Section 5. Finally, a conclusion will be drawn in Section 6.

\section{Preliminaries}

We compare a switching fuzzy control system and an SF control system.

2.1. Switching Fuzzy Control System. Consider a switching fuzzy model defined by Tanaka et al. as follows [16-19].

The Region Rule $j$ :

If $z_{1}(t)$ is $N_{j 1}$ and $\cdots$ and $z_{p}(t)$ is $N_{j p}$,

then the following is given.

The Local Region Rule $i$ :

$$
\begin{aligned}
& \text { If } z_{1}(t) \text { is } M_{j i 1} \text { and } \cdots \text { and } z_{p}(t) \text { is } M_{j i p} \text {, } \\
& \text { then } \\
& \qquad \dot{x}(t)=A_{j i} x(t)+B_{j i} u(t), \quad y(t)=C_{j i} x(t), \\
& \quad i=1,2, \ldots, r, \quad j=1,2, \ldots, s .
\end{aligned}
$$

$z_{1}(t), \ldots, z_{p}(t)$ are the premise variables. $M_{j i 1}, \ldots, M_{j i p}$ are the fuzzy sets. $r$ is the number of the if-then rules. This switching fuzzy model in [16-19] has two levels of structures: the region rule level and the local fuzzy rule level. The region rule is crisply switched according to the premise variables. $s$ is the number of regions partitioned on the premise variables space. Note that $N_{j k}(z(t))$ is a crisp set.

Where

$$
N_{j k}(z(t))= \begin{cases}1, & z(t) \in N_{j k} \\ 0, & \text { otherwise }\end{cases}
$$

When the variables $z_{1}(t), \ldots, z_{p}(t)$ satisfy the condition of the region rule, the fuzzy model which belongs to the local region rule below the region rule is active.

2.2. SF Control System. Comparing with the previous works [16-19], the SF model breaks through two levels of structures, but it is switching between each of the sub fuzzy models 


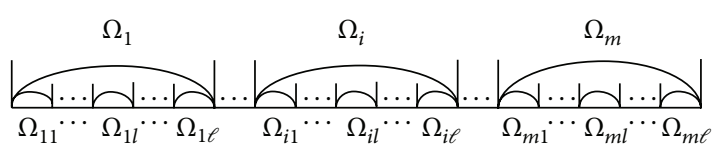

FIGURE 1: Sketch map of the switched fuzzy systems.

directly. The SF system is a more general system than the model of [16-19]. Now, we introduce the SF systems.

When subsystems of the switched systems are T-S fuzzy systems, the systems are switched fuzzy systems. Now, we define SF model including $N_{\sigma(t)}$ pieces of rules as follows:

$$
\begin{aligned}
& R_{\sigma(t)}^{l}: \text { if } \bar{x}_{\sigma(t) 1}(t) \text { is } M_{\sigma(t) 1}^{l}, \ldots, \text { and } \bar{x}_{\sigma(t) p}(t) \text { is } M_{\sigma(t) p}^{l} \text {, } \\
& \text { then }
\end{aligned}
$$$$
\dot{x}(t)=A_{\sigma(t) l} x(t)+B_{\sigma(t) l} u_{\sigma(t)}(t), \quad l=1,2, \ldots, N_{\sigma(t)},
$$

with

$$
\sigma(t): M=\{1,2, \ldots, m\}
$$

which is a piecewise constant function, called a switching signal.

$R_{\sigma(t)}^{l}$ denotes the $l$ th fuzzy inference rule, $N_{\sigma(t)}$ is the number of inference rules, $u_{\sigma(t)}(t)$ is the input variable, $x(t)$ is the state variable vector, $A_{\sigma(t) l}$ and $B_{\sigma(t) l}$ are matrices of appropriate dimensions and $\bar{x}_{\sigma(t) 1}, \bar{x}_{\sigma(t) 2}, \ldots, \bar{x}_{\sigma(t) p}$ are the vectors of premise variables.

Sketch map of the switched fuzzy systems is depicted in Figure $1 ; \Omega_{i}$ denotes the state area of the $i$ th switched subsystem; $\Omega_{i l}$ denote the $l$ th sub fuzzy area in $\Omega_{i}$. In fact, the switched fuzzy systems again partition the $\Omega_{i}$ subarea into $l$ sub fuzzy areas $\Omega_{i 1}, \ldots, \Omega_{i l}, \ldots, \Omega_{i \ell}$. There is local linear model in every sub fuzzy area; namely, local linear model in $\Omega_{i l}$ is $\dot{x}(t)=A_{i l} x(t)+B_{i l} u_{i}(t)$. The model of every switched subarea $\Omega_{1}, \ldots, \Omega_{m}$ is composed of local linear model which is linked by fuzzy membership function. We design the switching law for sub fuzzy area model to ensure stability of the switched fuzzy system. When local model in sub fuzzy area satisfies the switching law, we switch to the $\Omega_{i}$ th subsystem to ensure stability of the switched fuzzy system.

Also, switching law can be designed by arbitrary form, not only depending on fuzzy rules as in [16-19]. That is, the SF systems are extended further for the switching fuzzy systems (1) and (2).

\section{Novel Models of Uncertain Switched Fuzzy Time-Delay Systems}

So in here, we will introduce an innovated representation modeling of uncertain SF time-delay systems. In this model, each subsystem is an uncertain fuzzy time-delay system, namely, uncertain sub fuzzy time-delay system.
Consider the uncertain SF time-delay model including $N_{\sigma(t)}$ pieces of rules as follows:

$$
\begin{aligned}
& R_{\sigma(t)}^{l} \text { : if } \bar{x}_{\sigma(t) 1}(t) \text { is } M_{\sigma(t) 1}^{l}, \ldots \text {, and } \bar{x}_{\sigma(t) p}(t) \text { is } M_{\sigma(t) p}^{l} \text {, } \\
& \text { then } \\
& \dot{x}(t)=\left(A_{\sigma(t) l}+\Delta A_{\sigma(t) l}\right) x(t)+D_{\sigma(t) l} x(t-\tau) \\
& +B_{\sigma(t) l} u_{\sigma(t) l}(t)+\bar{D}_{\sigma(t)} \omega(t), \\
& y(t)=C_{\sigma(t) l} x(t), \quad t \in[0, \infty), \quad x(0)=0, \\
& \varphi_{\sigma(t)}(\theta)=x(t+\theta), \quad \theta \in\left[t_{j}-\tau, t_{j}\right], \\
& j=0,1, \ldots, \quad l=1,2, \ldots, N_{\sigma(t)},
\end{aligned}
$$

with

$$
\sigma(t): M=\{1,2, \ldots, m\}
$$

which is a piecewise constant function, called a switching signal. It can be characterized by the switching sequence $\sum=\left\{x_{0} ;\left(i_{0}, t_{0}\right),\left(i_{1}, t_{1}\right), \ldots,\left(i_{j}, t_{j}\right) \quad \mid \quad i_{j} \in M, j=\right.$ $0,1, \ldots\}$. Moreover, $\sigma(t)=i$ implies that the $i$ th subsystem $\left(A_{i}, D_{i}, B_{i}, C_{i}\right)$ is active.

$R_{\sigma(t)}^{l}$ denotes the $l$ th fuzzy inference rule, $N_{\sigma(t)}$ is the number of inference rules, $u_{\sigma(t) l}(t) \in \mathbb{R}^{q}$ is the control input, $x(t) \in \mathbb{R}^{n}$ and $y(t) \in \mathbb{R}^{s}$ denote the state vector and the output vector, respectively, $\omega(t) \in \mathbb{R}^{n}$ is the bounded exogenous disturbance, $\bar{D}_{\sigma(t) l}$ are known constant matrices of appropriate dimensions, $A_{\sigma(t) l}$ and $D_{\sigma(t) l} \in \mathbb{R}^{n \times n}$ are known system matrices, and $B_{\sigma(t) l} \in \mathbb{R}^{n \times q}$ is the input matrix. Moreover; $C_{\sigma(t) l} \in \mathbb{R}^{s \times n}$ is the output matrix, $\tau$ is the constantbounded time delay in the state, and $\varphi_{\sigma(t)}(t)$ is the continuous vector-valued function.

It is readily seen that the $i$ th sub fuzzy system can be represented as follows:

$$
\begin{aligned}
& R_{i}^{l}: \text { if } \bar{x}_{i 1}(t) \text { is } M_{i 1}^{l}, \ldots, \text { and } \bar{x}_{i p}(t) \text { is } M_{i p}^{l}, \\
& \text { then } \\
& \begin{aligned}
& \dot{x}(t)=\left(A_{i l}+\Delta A_{i l}\right) x(t)+D_{i l} x(t-\tau) \\
&+B_{i l} u_{i l}(t)+\bar{D}_{i} \omega(t), \\
& y(t)= C_{i l} x(t), \quad t \in[0, \infty), \quad x(0)=0, \\
& \quad l=1,2, \ldots, N_{i}, \quad i=1,2, \ldots, m .
\end{aligned}
\end{aligned}
$$

Therefore, the global model of the $i$ th sub fuzzy system is described by means of the following equation:

$$
\begin{aligned}
\dot{x}(t)=\sum_{l=1}^{N_{i}} \eta_{i l}(\bar{x}(t))[ & \left(A_{i l}+\Delta A_{i l}\right) x(t) \\
& \left.+D_{i l} x(t-\tau)+B_{i l} u_{i l}(t)+\bar{D}_{i} \omega(t)\right], \\
y(t) & =\sum_{l=1}^{N_{i}} \eta_{i l} C_{i l} x(t),
\end{aligned}
$$


along with

$$
\begin{gathered}
0 \leq \eta_{i l}(\bar{x}(t)) \leq 1, \quad \sum_{l=1}^{N_{i}} \eta_{i l}(\bar{x}(t))=1, \\
w_{i l}(\bar{x}(t))=\prod_{\rho=1}^{p} M_{i \rho}^{l}\left(\bar{x}_{\rho}(t)\right), \quad \eta_{i l}(\bar{x}(t))=\frac{w_{i l}(\bar{x}(t))}{\sum_{l=1}^{N_{i}} w_{i l}(\bar{x}(t))},
\end{gathered}
$$

where $M_{i \rho}^{l}\left(\bar{x}_{\rho}(t)\right)$ denotes the membership function in which $\bar{x}_{\rho}(t)$ belongs to the fuzzy set $M_{i \rho}^{l}$.

\section{New Stabilization Based on State Observer and Combined Switching Controller}

Suppose that the state observer is given by the following:

$$
\begin{aligned}
& R_{\sigma(t)}^{l} \text { : if } \bar{x}_{\sigma(t) 1}(t) \text { is } M_{\sigma(t) 1}^{l}, \ldots \text {, and } \bar{x}_{\sigma(t) p}(t) \text { is } M_{\sigma(t) p}^{l}, \\
& \text { then } \\
& \dot{\hat{x}}(t)=A_{\sigma(t) l} \widehat{x}(t)+D_{\sigma(t) l} \widehat{x}(t-\tau)+B_{\sigma(t) l} u_{\sigma(t) l}(t) \\
& +\bar{D}_{\sigma(t)} \omega(t)+L_{\sigma(t) l}(y(t)-\widehat{y}(t)), \\
& \widehat{y}(t)=C_{\sigma(t) l} \widehat{x}(t)
\end{aligned}
$$

in which $y(t)$ and $\sigma(t)$ are measurable output and switching signal of systems (5), respectively. The matrices $L_{\sigma(t) l} \in \mathbb{R}^{n \times s}$ are to be determined later.

Then, the global model of the $i$ th sub fuzzy observer is described as follows:

$$
\begin{aligned}
& \dot{\hat{x}}(t)=\sum_{l=1}^{N_{i}} \eta_{i l}(\bar{x}(t))\left[A_{i l} \widehat{x}(t)+D_{i l} \widehat{x}(t-\tau)\right. \\
&+B_{i l} u_{i l}(t)+\bar{D}_{i} \omega(t) \\
&\left.+L_{i l}(y(t)-\widehat{y}(t))\right], \\
& \hat{y}(t)=\sum_{l=1}^{N_{i}} \eta_{i l}(\bar{x}(t)) C_{i l} \widehat{x}(t) .
\end{aligned}
$$

Define the difference between the real state and the observer state as follows:

$$
e(t)=x(t)-\widehat{x}(t)
$$

Subtracting (11) from (8) gives the following error switched system:

$$
\begin{array}{r}
\dot{e}(t)=\sum_{l=1}^{N_{i}} \eta_{i l} \sum_{h=1}^{N_{i}} \eta_{i h}[ \\
\left.+\Delta A_{i l}+\Delta A_{i l}-L_{i l} C_{i h}\right) e(t) \\
\left.+D_{i l} e(t-\tau)\right] .
\end{array}
$$

Our purpose is to design a controller $u=u(t)$ and a switching law $\sigma=\sigma(t)$ such that (5) is asymptotically stable for the observer-based robust control.

Now, a combined switching controller is employed to control the SF time-delay model of (5). The combined switching controller, which has nonfuzzy rules, integrates the advantages of both switching controllers and supplementary controllers but eliminates their disadvantages. The switching controllers consist of some simple subcontrollers. These subcontrollers will switch among each other to control the system of (5) according to an appropriate switching scheme. The supplementary controller is employed to handle the external disturbance. It is shown in the sequel how to design controllers to achieve asymptotical stability in the closed loop and under the switching law.

The combined switching controller for the sub fuzzy timedelay system is described by

$$
u_{i l}=u_{i l}^{1}+u_{i l}^{2}
$$

where $u_{i l}^{1}$ is the switching controller and $u_{i l}^{2}$ is the supplementary controller. Consider the following:

$$
\begin{gathered}
u_{i l}^{1}=-\sum_{a=1}^{N_{i}} \rho_{i l a}(\widehat{x}(t)) R B_{i a}^{T} P_{i} \hat{x}(t), \\
u_{i l}^{2}= \begin{cases}0, & \text { if }\left\|B_{i l}^{T} P_{i} \hat{x}(t)\right\|=0, \\
\Theta_{i l}, & \text { otherwise, }\end{cases} \\
\Theta_{i l}=-\frac{\theta(t) \cdot B_{i l}^{T} P_{i} \widehat{x}(t)}{\left\|B_{i l}^{T} P_{i} \widehat{x}(t)\right\|} \\
-\sum_{\beta=1}^{N_{i}} \frac{\gamma_{\beta} \theta(t) \cdot B_{i l}^{T} P_{i} \hat{x}(t)}{\left\|B_{i l}^{T} P_{i} \hat{x}(t)\right\|^{2}}\left\|B_{i \beta}^{T} P_{i} \widehat{x}(t)\right\|,
\end{gathered}
$$

where $R$ is symmetric positive definite.

Now, we will make the following standard assumptions for the uncertain SF system (5).

Assumption 1. The admissible parameter uncertainties are of the norm-bounded form

$$
\Delta A_{i l}=M_{i l} F_{i l}(t) N_{i l},
$$

where $M_{i l}$ and $N_{i l}$ are known real constant matrices of proper dimensions which represent the structure of the uncertainties and $F_{i l}(t)$ is unknown time-varying matrix satisfying

$$
F_{i l}^{T}(t) F_{i l}(t) \leq I .
$$

Assumption 2. There exists constant $\gamma_{l}\left(l=1,2, \ldots N_{i}\right)$, such that

$$
\bar{D}_{i}=\sum_{l=1}^{N_{i}} \gamma_{l} B_{i l} .
$$

Assumption 3. There exists known continuous nonnegative function $\theta(t)$, such that

$$
\max \|\omega(t)\| \leq \theta(t) .
$$


The following theorem provides a condition for asymptotic stability of the system (5) and gives the design of statedependent switching laws.

Theorem 4. Suppose that there exist constants $\beta_{i j}(i, j \in \bar{M})$ (either all nonnegative or all nonpositive) and positive definite symmetric matrices $P_{i}, Q_{i}$ such that the following matrix inequalities are satisfied. Then, the system (5) along with fuzzy observer (10) is asymptotical stable via the combined switching controller (14):

$$
\begin{aligned}
& {\left[\begin{array}{cccc}
\Upsilon_{i a l}+\sum_{j=1}^{m} \beta_{i j}\left(P_{i}-P_{j}\right) & P_{i} D_{i l} & P_{i} L_{i l} C_{i h} & 0 \\
D_{i l}^{T} P_{i} & -P_{i} & 0 & 0 \\
C_{i h}^{T} L_{i l}^{T} P_{i} & 0 & \Psi_{i l h} & Q_{i} D_{i l} \\
0 & 0 & D_{i l}^{T} Q_{i} & -Q_{i}
\end{array}\right]<0,} \\
& i=1,2, \ldots, m, \quad l, a, h=1,2, \ldots N_{i} \text {, }
\end{aligned}
$$

where $\Upsilon_{i a l}=-P_{i} B_{i a} R B_{i l}^{T} P_{i}-P_{i} B_{i l} R B_{i a}^{T} P_{i}+A_{i l}^{T} P_{i}+P_{i} A_{i l}+N_{i l}^{T} N_{i l}+$ $P_{i}$,

$$
\begin{aligned}
\Psi_{i l h}= & Q_{i}+\left(A_{i l}^{T}-C_{i h}^{T} L_{i l}^{T}\right) Q_{i}+Q_{i}\left(A_{i l}-L_{i l} C_{i h}\right) \\
& +2 Q_{i} M_{i l} M_{i l}^{T} Q_{i}+N_{i l}^{T} N_{i l} .
\end{aligned}
$$

And, the switching law is designed as follows:

$$
\sigma=\sigma(\widehat{x}(t))=i:[0,+\infty) \longrightarrow \bar{M}=\{1,2, \ldots, m\} .
$$

Proof. Without loss of generality, suppose that $\beta_{i j} \geq 0$. For any $i \in \bar{M}$, if $\widehat{x}^{T}(t)\left(P_{i}-P_{j}\right) \widehat{x}(t) \geq 0$, for all $j \in \bar{M}$, then from (21), we have

$$
\left[\begin{array}{cccc}
\Upsilon_{i a l} & P_{i} D_{i l} & P_{i} L_{i l} C_{i h} & 0 \\
D_{i l}^{T} P_{i} & -P_{i} & 0 & 0 \\
C_{i h}^{T} L_{i l}^{T} P_{i} & 0 & \Psi_{i l h} & Q_{i} D_{i l} \\
0 & 0 & D_{i l}^{T} Q_{i} & -Q_{i}
\end{array}\right]<0
$$

Obviously, for all $\widehat{x}(t) \in \mathbb{R}^{n} \backslash\{0\}$, there is certainly an $i \in \bar{M}$ such that

$$
\widehat{x}^{T}(t)\left(P_{i}-P_{j}\right) \widehat{x}(t) \geq 0, \quad \forall j \in \bar{M} .
$$

For arbitrary $i \in \bar{M}$, let

$$
\Omega_{i}=\left\{\widehat{x}(t) \in R^{n} \backslash\{0\} \mid \widehat{x}^{T}(t)\left(P_{i}-P_{j}\right) \widehat{x}(t) \geq 0, \forall j \in \bar{M}\right\} .
$$

Then, $\bigcup_{i=1}^{m} \Omega_{i}=\mathbb{R}^{n} \backslash\{0\}$. Furthermore, let us construct the sets $\bar{\Omega}_{1}=\Omega_{1}, \ldots, \bar{\Omega}_{i}=\Omega_{i}-\bigcup_{j=1}^{i-1} \bar{\Omega}_{j}, \ldots, \bar{\Omega}_{m}=\Omega_{m}-$ $\bigcup_{j=1}^{m-1} \bar{\Omega}_{j}$. Obviously, we have $\bigcup_{i=1}^{m} \Omega_{i}=\mathbb{R}^{n} \backslash\{0\}$ and $\bar{\Omega}_{i} \cap \bar{\Omega}_{j}=$ $\Phi, i \neq j$.
Note that (24) holds; then there exists

$$
\Xi^{T}(t)\left[\begin{array}{cccc}
\Upsilon_{i a l} & P_{i} D_{i l} & P_{i} L_{i l} C_{i h} & 0 \\
D_{i l}^{T} P_{i} & -P_{i} & 0 & 0 \\
C_{i h}^{T} L_{i l}^{T} P_{i} & 0 & \Psi_{i l h} & Q_{i} D_{i l} \\
0 & 0 & D_{i l}^{T} Q_{i} & -Q_{i}
\end{array}\right] \Xi(t)<0,
$$

where $\Xi(t) \triangleq\left[\begin{array}{llll}\hat{x}^{T}(t) & \hat{x}^{T}(t-\tau) & e^{T}(t) & e^{T}(t-\tau)\end{array}\right]^{T}$.

When $\widehat{x}(t) \in \bar{\Omega}_{i}, \sigma(t)=i$, then choose the multiple Lyapunov functional as follows:

$$
\begin{aligned}
V_{i}(t)= & \widehat{x}^{T}(t) P_{i} \widehat{x}(t)+e^{T}(t) Q_{i} e(t) \\
& +\int_{t-\tau}^{t} \widehat{x}^{T}(\theta) P_{i} \widehat{x}(\theta) d \theta+\int_{t-\tau}^{t} e^{T}(\theta) Q_{i} e(\theta) d \theta,
\end{aligned}
$$

which is positive definite since $P_{i}$ and $Q_{i}$ are positive definite matrices.

The time derivative of $V_{i}(t)$ is given as follows:

$$
\begin{aligned}
& \dot{V}_{i}(t)=\dot{\hat{x}}^{T}(t) P_{i} \widehat{x}(t)+\widehat{x}^{T}(t) P_{i} \dot{\hat{x}}(t)+\dot{e}^{T}(t) Q_{i} e(t) \\
& +e^{T}(t) Q_{i} \dot{e}(t)+\widehat{x}^{T}(t) P_{i} \widehat{x}(t)-\widehat{x}^{T}(t-\tau) P_{i} \widehat{x}(t-\tau) \\
& +e^{T}(t) Q_{i} e(t)-e^{T}(t-\tau) Q_{i} e(t-\tau) \\
& =\sum_{l=1}^{N_{i}} \sum_{h=1}^{N_{i}} \eta_{i l} \eta_{i h}\left[A_{i l} \widehat{x}(t)+D_{i l} \widehat{x}(t-\tau)+B_{i l} u_{i l}(t)\right. \\
& \left.+\bar{D}_{i} \omega+L_{i l} C_{i h} e(t)\right]^{T} P_{i} \widehat{x}(t) \\
& +\sum_{l=1}^{N_{i}} \sum_{h=1}^{N_{i}} \eta_{i l} \eta_{i h} \widehat{x}^{T}(t) P_{i}\left[A_{i l} \widehat{x}(t)+D_{i l} \widehat{x}(t-\tau)\right. \\
& \left.+B_{i l} u_{i l}(t)+\bar{D}_{i} \omega+L_{i l} C_{i h} e(t)\right] \\
& +\sum_{l=1}^{N_{i}} \sum_{h=1}^{N_{i}} \eta_{i l} \eta_{i h}\left[\left(A_{i l}+\Delta A_{i l}-L_{i l} C_{i h}\right) e(t)\right. \\
& \left.+\Delta A_{i l} \widehat{x}(t)+D_{i l} e(t-\tau)\right]^{T} Q_{i} e(t) \\
& +\sum_{l=1}^{N_{i}} \sum_{h=1}^{N_{i}} \eta_{i l} \eta_{i h} e^{T}(t) Q_{i}\left[\left(A_{i l}+\Delta A_{i l}-L_{i l} C_{i h}\right) e(t)\right. \\
& \left.+\Delta A_{i l} \widehat{x}(t)+D_{i l} e(t-\tau)\right] \\
& +\widehat{x}^{T}(t) P_{i} \widehat{x}(t)-\widehat{x}^{T}(t-\tau) P_{i} \widehat{x}(t-\tau) \\
& +e^{T}(t) Q_{i} e(t)-e^{T}(t-\tau) Q_{i} e(t-\tau) \\
& =\sum_{l=1}^{N_{i}} \sum_{h=1}^{N_{i}} \eta_{i l} \eta_{i h}\left[\widehat{x}^{T}(t) A_{i l}^{T}+\widehat{x}^{T}(t-\tau) D_{i l}^{T}\right. \\
& \left.+u_{i l}^{T}(t) B_{i l}^{T}+\omega^{T} \bar{D}_{i}^{T}+e^{T}(t) C_{i h}^{T} L_{i l}^{T}\right] P_{i} \widehat{x}(t)
\end{aligned}
$$




$$
\begin{aligned}
& +\sum_{l=1}^{N_{i}} \sum_{h=1}^{N_{i}} \eta_{i l} \eta_{i h} \widehat{x}^{T}(t) P_{i}\left[A_{i l} \widehat{x}(t)+D_{i l} \widehat{x}(t-\tau)\right. \\
& \left.+B_{i l} u_{i l}(t)+\bar{D}_{i} \omega+L_{i l} C_{i h} e(t)\right] \\
& +\sum_{l=1}^{N_{i}} \sum_{h=1}^{N_{i}} \eta_{i l} \eta_{i h}\left[e^{T}(t)\left(A_{i l}^{T}+\Delta A_{i l}^{T}-C_{i h}^{T} L_{i l}^{T}\right)\right. \\
& \left.+\widehat{x}^{T}(t) \Delta A_{i l}^{T}+e^{T}(t-\tau) D_{i l}^{T}\right] Q_{i} e(t) \\
& +\sum_{l=1}^{N_{i}} \sum_{h=1}^{N_{i}} \eta_{i l} \eta_{i h} e^{T}(t) Q_{i}\left[\left(A_{i l}+\Delta A_{i l}-L_{i l} C_{i h}\right) e(t)\right. \\
& \left.+\Delta A_{i l} \widehat{x}(t)+D_{i l} e(t-\tau)\right] \\
& +\widehat{x}^{T}(t) P_{i} \widehat{x}(t)-\widehat{x}^{T}(t-\tau) P_{i} \widehat{x}(t-\tau) \\
& +e^{T}(t) Q_{i} e(t)-e^{T}(t-\tau) Q_{i} e(t-\tau) \\
& =\sum_{l=1}^{N_{i}} \sum_{h=1}^{N_{i}} \eta_{i l} \eta_{i h}\left[\widehat{x}^{T}(t)\left(A_{i l}^{T} P_{i}+P_{i} A_{i l}\right) \widehat{x}(t)\right. \\
& +u_{i l}^{T}(t) B_{i l}^{T} P_{i} \widehat{x}(t) \\
& +\widehat{x}^{T}(t) P_{i} B_{i l} u_{i l}(t)+\widehat{x}^{T}(t-\tau) D_{i l}^{T} P_{i} \widehat{x}(t) \\
& +\widehat{x}^{T}(t) P_{i} D_{i l} \widehat{x}(t-\tau)+\omega^{T} \bar{D}_{i}^{T} P_{i} \widehat{x}(t) \\
& +\widehat{x}^{T}(t) P_{i} \bar{D}_{i} \omega+e^{T}(t) C_{i h}^{T} L_{i l}^{T} P_{i} \widehat{x}(t) \\
& \left.+\widehat{x}^{T}(t) P_{i} L_{i l} C_{i h} e(t)\right] \\
& +\sum_{l=1}^{N_{i}} \sum_{h=1}^{N_{i}} \eta_{i l} \eta_{i h}\left[\widehat{x}^{T}(t) \Delta A_{i l}^{T} Q_{i} e(t)+e^{T}(t) Q_{i} \Delta A_{i l} \widehat{x}(t)\right. \\
& +e^{T}(t)\left(A_{i l}^{T}+\Delta A_{i l}^{T}-C_{i h}^{T} L_{i l}^{T}\right) Q_{i} e(t) \\
& +e^{T}(t) Q_{i}\left(A_{i l}+\Delta A_{i l}-L_{i l} C_{i h}\right) e(t) \\
& +e^{T}(t-\tau) D_{i l}^{T} Q_{i} e(t) \\
& \left.+e^{T}(t) Q_{i} D_{i l} e(t-\tau)\right] \\
& +\widehat{x}^{T}(t) P_{i} \widehat{x}(t)-\widehat{x}^{T}(t-\tau) P_{i} \widehat{x}(t-\tau) \\
& +e^{T}(t) Q_{i} e(t)-e^{T}(t-\tau) Q_{i} e(t-\tau) \text {. }
\end{aligned}
$$

(I) When $B_{i l}^{T} P_{i} \widehat{x}(t) \neq 0$, then we have

$$
\begin{aligned}
\hat{x}^{T}(t)( & \left.A_{i l}^{T} P_{i}+P_{i} A_{i l}\right) \hat{x}(t)+\left(u_{i l}^{1}\right)^{T}(t) B_{i l}^{T} P_{i} \hat{x}(t) \\
& +\widehat{x}^{T}(t) P_{i} B_{i l} u_{i l}^{1}(t) \\
= & \hat{x}^{T}(t)\left[\left(-\sum_{a=1}^{N_{i}} \rho_{i l a} R B_{i a}^{T} P_{i}\right)^{T} B_{i l}^{T} P_{i}\right.
\end{aligned}
$$

$$
\begin{aligned}
& +P_{i} B_{i l}\left(-\sum_{a=1}^{N_{i}} \rho_{i l a} R B_{i a}^{T} P_{i}\right) \\
& \left.+A_{i l}^{T} P_{i}+P_{i} A_{i l}\right] \widehat{x}(t) \\
& =\widehat{x}^{T}(t)\left\{\left[\left(-\sum_{a=1}^{N_{i}} \rho_{i l a} R B_{i a}^{T} P_{i}\right)^{T} B_{i l}^{T} P_{i}\right.\right. \\
& -\sum_{a=1}^{N_{i}} \eta_{i a}\left(B_{i l} R B_{i a}^{T} P_{i}\right)^{T} P_{i} \\
& \left.+\sum_{a=1}^{N_{i}} \eta_{i a}\left(B_{i l} R B_{i a}^{T} P_{i}\right)^{T} P_{i}\right] \\
& +P_{i} B_{i l}\left(-\sum_{a=1}^{N_{i}} \rho_{i l a} R B_{i a}^{T} P_{i}\right) \\
& -\sum_{a=1}^{N_{i}} \eta_{i a} P_{i}\left(B_{i l} R B_{i a}^{T} P_{i}\right) \\
& \left.+\sum_{a=1}^{N_{i}} \eta_{i a} P_{i}\left(B_{i l} R B_{i a}^{T} P_{i}\right)+A_{i l}^{T} P_{i}+P_{i} A_{i l}\right\} \widehat{x}(t) \\
& =\widehat{x}^{T}(t)\left\{-\sum_{a=1}^{N_{i}} \eta_{i a}\left(B_{i l} R B_{i a}^{T} P_{i}\right)^{T} P_{i}-\sum_{a=1}^{N_{i}}\left(\rho_{i l a}-\eta_{i a}\right)\right. \\
& \times\left(B_{i l} R B_{i a}^{T} P_{i}\right)^{T} P_{i} \\
& -\sum_{a=1}^{N_{i}} \eta_{i a} P_{i}\left(B_{i l} R B_{i a}^{T} P_{i}\right)-\sum_{a=1}^{N_{i}}\left(\rho_{i l a}-\eta_{i a}\right) \\
& \times P_{i}\left(B_{i l} R B_{i a}^{T} P_{i}\right) \\
& \left.+A_{i l}^{T} P_{i}+P_{i} A_{i l}\right\} \hat{x}(t)
\end{aligned}
$$

Let

$$
\begin{gathered}
\rho_{i l a}=\frac{1+\operatorname{sgn}\left(\widehat{x}^{T}(t) P_{i} B_{i l} R B_{i a}^{T} P_{i} \widehat{x}(t)\right)}{2}, \\
\operatorname{sgn}(z)= \begin{cases}1, & \text { if } z>0, \\
-1, & \text { otherwise. }\end{cases}
\end{gathered}
$$

From (30), we obtain

$$
\begin{aligned}
=\widehat{x}^{T}(t)\left\{\sum_{a=1}^{N_{i}} \eta_{i a}(\right. & -P_{i} B_{i a} R B_{i l}^{T} P_{i}-P_{i} B_{i l} R B_{i a}^{T} P_{i} \\
& \left.+A_{i l}^{T} P_{i}+P_{i} A_{i l}\right) \\
-2 \sum_{a=1}^{N_{i}}\left(\frac{\operatorname{sgn}\left(e_{r}^{T}(t) P_{i} B_{i l} R B_{i a}^{T} P_{i} e_{r}(t)\right)}{2}\right. & \left.\left.-\left(\eta_{i a}-\frac{1}{2}\right)\right)\left(B_{i l} R B_{i a}^{T} P_{i}\right)^{T} P_{i}\right\} \widehat{x}(t)
\end{aligned}
$$




$$
\begin{gathered}
\leq \sum_{a=1}^{N_{i}} \eta_{i a} \hat{x}^{T}(t)\left(-P_{i} B_{i a} R B_{i l}^{T} P_{i}-P_{i} B_{i l} R B_{i a}^{T} P_{i}\right. \\
\left.+A_{i l}^{T} P_{i}+P_{i} A_{i l}\right) \hat{x}(t) \\
-2 \sum_{a=1}^{N_{i}}\left(\frac{1}{2}-\left(\eta_{i a}-\frac{1}{2}\right)\right)\left|\widehat{x}^{T}(t) P_{i} B_{i l} R B_{i a}^{T} P_{i} \hat{x}(t)\right|
\end{gathered}
$$

As $\eta_{i a}-(1 / 2) \in\left[\begin{array}{ll}-1 / 2 & 1 / 2\end{array}\right]$, due to the property of the switched fuzzy model with time delay, it can be shown that (33) satisfies the following inequality:

$$
\begin{array}{r}
\widehat{x}^{T}(t)\left(A_{i l}^{T} P_{i}+P_{i} A_{i l}\right) \hat{x}(t)+\left(u_{i l}^{1}\right)^{T}(t) B_{i l}^{T} P_{i} \widehat{x}(t) \\
+\widehat{x}^{T}(t) P_{i} B_{i l} u_{i l}^{1}(t) \\
\leq \sum_{a=1}^{N_{i}} \eta_{i a} \widehat{x}^{T}(t)\left(-P_{i} B_{i a} R B_{i l}^{T} P_{i}-P_{i} B_{i l} R B_{i a}^{T} P_{i}\right. \\
\left.+A_{i l}^{T} P_{i}+P_{i} A_{i l}\right) \hat{x}(t) .
\end{array}
$$

Considering Assumptions 2 and 3, we have

$$
\begin{aligned}
& \omega^{T} \bar{D}_{i}^{T} P \widehat{x}(t)+\widehat{x}^{T}(t) P \bar{D}_{i} \omega+\left(u_{i l}^{2}\right)^{T}(t) B_{i l}^{T} P \widehat{x}(t) \\
& +\widehat{x}^{T}(t) P B_{i l} u_{i l}^{2}(t) \\
& =2\left(\hat{x}^{T}(t) P_{i} B_{i l} u_{i l}^{2}+\widehat{x}^{T}(t) P_{i} \bar{D}_{i} \omega\right) \\
& =2 \widehat{x}^{T}(t) P_{i} B_{i l}\left[-\frac{\theta(t)\left(B_{i l}^{T} P_{i} \widehat{x}(t)\right)}{\left\|B_{i l}^{T} P_{i} \widehat{x}(t)\right\|}\right. \\
& \left.-\sum_{\beta=1}^{N_{i}} \frac{\gamma_{\beta} \theta(t)\left(B_{i l}^{T} P_{i} \hat{x}(t)\right)}{\left\|B_{i l}^{T} P_{i} \hat{x}(t)\right\|^{2}} \cdot\left\|B_{i \beta}^{T} P_{i} \widehat{x}(t)\right\|\right] \\
& +2\left[\widehat{x}^{T}(t) P_{i}\left(\sum_{\beta=1}^{N_{i}} \gamma_{\beta} B_{i \beta}\right) \omega\right] \\
& \leq 2\left(-\theta(t)\left\|B_{i l}^{T} P_{i} \widehat{x}(t)\right\|-\sum_{\beta=1}^{N_{i}} \gamma_{\beta} \theta(t)\left\|B_{i \beta}^{T} P_{i} \widehat{x}(t)\right\|\right) \\
& +2 \sum_{\beta=1}^{N_{i}} \gamma_{\beta} \theta(t)\left\|B_{i \beta}^{T} P_{i} \widehat{x}(t)\right\| \\
& =2\left(-\theta(t)\left\|B_{i l}^{T} P_{i} \widehat{x}(t)\right\|\right) .
\end{aligned}
$$

According to Assumption 1, we have

$$
\begin{aligned}
\hat{x}^{T}(t) \Delta A_{i l}^{T} Q_{i} e(t)+e^{T}(t) Q_{i} \Delta A_{i l} \widehat{x}(t)+e^{T}(t) \Delta A_{i l}^{T} Q_{i} e(t) \\
+e^{T}(t) Q_{i} \Delta A_{i l} e(t) \leq \widehat{x}^{T}(t) N_{i l}^{T} N_{i l} \widehat{x}(t) \\
+2 e^{T}(t) Q_{i} M_{i l} M_{i l}^{T} Q_{i} e(t) \\
+e^{T}(t) N_{i l}^{T} N_{i l} e(t)
\end{aligned}
$$

Then, from (34)-(36), it holds true that

$$
\begin{aligned}
& \dot{V}(t) \leq \sum_{l=1}^{N_{i}} \sum_{h=1}^{N_{i}} \sum_{a=1}^{N_{i}} \eta_{i l} \eta_{i h} \eta_{i a} \\
& \times\left[\widehat { x } ^ { T } ( t ) \left(-P_{i} B_{i a} R B_{i l}^{T} P_{i}-P_{i} B_{i l} R B_{i a}^{T} P_{i}\right.\right. \\
& \left.+A_{i l}^{T} P_{i}+P_{i} A_{i l}\right) \widehat{x}(t) \\
& +\widehat{x}^{T}(t-\tau) D_{i l}^{T} P_{i} \widehat{x}(t) \\
& +\widehat{x}^{T}(t) P_{i} D_{i l} \widehat{x}(t-\tau) \\
& +e^{T}(t) C_{i h}^{T} L_{i l}^{T} P_{i} \widehat{x}(t) \\
& +\widehat{x}^{T}(t) P_{i} L_{i l} C_{i h} e(t) \\
& +\widehat{x}^{T}(t) N_{i l}^{T} N_{i l} \widehat{x}(t) \\
& +2 e^{T}(t) Q_{i} M_{i l} M_{i l}^{T} Q_{i} e(t) \\
& +e^{T}(t) N_{i l}^{T} N_{i l} e(t) \\
& +e^{T}(t)\left(A_{i l}^{T}-C_{i h}^{T} L_{i l}^{T}\right) Q_{i} e(t) \\
& +e^{T}(t) Q_{i}\left(A_{i l}-L_{i l} C_{i h}\right) e(t) \\
& +e^{T}(t-\tau) D_{i l}^{T} Q_{i} e(t) \\
& +e^{T}(t) Q_{i} D_{i l} e(t-\tau) \\
& +\widehat{x}^{T}(t) P_{i} \widehat{x}(t)-\widehat{x}^{T}(t-\tau) P_{i} \widehat{x}(t-\tau) \\
& \left.+e^{T}(t) Q_{i} e(t)-e^{T}(t-\tau) Q_{i} e(t-\tau)\right] \\
& =\sum_{l=1}^{N_{i}} \sum_{h=1}^{N_{i}} \sum_{a=1}^{N_{i}} \eta_{i l} \eta_{i h} \eta_{i a}\left[\begin{array}{c}
\widehat{x}(t) \\
\widehat{x}(t-\tau) \\
e(t) \\
e(t-\tau)
\end{array}\right]^{T} \\
& \times\left[\begin{array}{cccc}
\Upsilon_{i a l} & P_{i} D_{i l} & P_{i} L_{i l} C_{i h} & 0 \\
D_{i l}^{T} P_{i} & -P_{i} & 0 & 0 \\
C_{i h}^{T} L_{i l}^{T} P_{i} & 0 & \Psi_{i l h} & Q_{i} D_{i l} \\
0 & 0 & D_{i l}^{T} Q_{i} & -Q_{i}
\end{array}\right] \\
& \times\left[\begin{array}{c}
\widehat{x}(t) \\
\widehat{x}(t-\tau) \\
e(t) \\
e(t-\tau)
\end{array}\right]
\end{aligned}
$$


where

$$
\begin{aligned}
\Upsilon_{i a l}= & -P_{i} B_{i a} R B_{i l}^{T} P_{i}-P_{i} B_{i l} R B_{i a}^{T} P_{i}+A_{i l}^{T} P_{i} \\
& +P_{i} A_{i l}+N_{i l}^{T} N_{i l}+P_{i}, \\
\Psi_{i l h}= & Q_{i}+\left(A_{i l}^{T}-C_{i h}^{T} L_{i l}^{T}\right) Q_{i}+Q_{i}\left(A_{i l}-L_{i l} C_{i h}\right) \\
& +2 Q_{i} M_{i l} M_{i l}^{T} Q_{i}+N_{i l}^{T} N_{i l},
\end{aligned}
$$

in which $i=\sigma(t)$ given by (23). Taking (9), (27), and (37) into account, we deduce that $(d / d t) V(t)<0, \Xi(t) \neq 0$. Therefore, the system (5) is asymptotical stable under the switching law (23).

(II) When $B_{i l}^{T} P_{i} \widehat{x}(t)=0$, then we have

$$
\begin{aligned}
\dot{V}_{i}(t) \leq \sum_{l=1}^{N_{i}} \sum_{h=1}^{N_{i}} \sum_{a=1}^{N_{i}} \eta_{i l} \eta_{i h} \eta_{i a}\left[\begin{array}{c}
\widehat{x}(t) \\
\hat{x}(t-\tau) \\
e(t) \\
e(t-\tau)
\end{array}\right]^{T} \\
\times \\
\times\left[\begin{array}{cccc}
\Upsilon_{i a l} & P_{i} D_{i l} & P_{i} L_{i l} C_{i h} & 0 \\
D_{i l}^{T} P_{i} & -P_{i} & 0 & 0 \\
C_{i h}^{T} L_{i l}^{T} P_{i} & 0 & \Psi_{i l h} & Q_{i} D_{i l} \\
0 & 0 & D_{i l}^{T} Q_{i} & -Q_{i}
\end{array}\right] \\
\times\left[\begin{array}{c}
\hat{x}(t) \\
\hat{x}(t-\tau) \\
e(t) \\
e(t-\tau)
\end{array}\right] .
\end{aligned}
$$

Taking (9) and (21) into account, we deduce that $(d / d t) V_{i}(x(t))<0, x(t) \neq 0$. Therefore, asymptotic stability follows.

Remark 5. Although any individual subsystem of (5) alone usually cannot achieve the observer-based robust control, a suitable switching may solve the robust control problem, as shown in Theorem 4.

Remark 6. (i) This controller structure consists of two parts: the switching controller $u_{i l}^{1}$ to stabilize the nominal system and the supplementary controller $u_{i l}^{2}$ to handle the external disturbance. Hence, in practice, this controller is a hybrid switching-robust controller. (ii) It can be seen from (15) that the switching controller $u_{i l}^{1}$ consists of $2^{N_{i}}$ controllers which are linear combinations of $-R B_{i a}^{T} P_{i} e_{r}(t), a=1,2, \ldots, N_{i}$.

In other words, we formulate the finding of $P_{i}$ and $Q_{i}$ of (21) into LMI problem. Considering (21) and multiplying both sides of (21) by the matrix $\operatorname{diag}\left\{P_{i}^{-1}, I, I, I\right\}$, we restate as follows.

Theorem 7. Suppose that there exist constants $\beta_{i j}(i, j \in \bar{M})$ (either all nonnegative or all nonpositive) and positive definite symmetric matrices $P_{i}, Q_{i}$ and matrices $G_{i l}$ such that the following matrix inequalities are satisfied. Then, the system (5) along with the fuzzy observer (10) is asymptotically stable via the combined switching controller (14), and the switching law is designed as (23):

$$
\begin{aligned}
& {\left[\begin{array}{cccccc}
\bar{\Upsilon}_{i a l}+\sum_{j=1}^{m} \beta_{i j}\left(P_{i}-P_{j}\right) & P_{i}^{-1} N_{i l}^{T} & D_{i l} & L_{i l} C_{i h} & 0 & 0 \\
* & -I & 0 & 0 & 0 & 0 \\
* & * & -P_{i} & 0 & 0 & 0 \\
* & * & * & \bar{\Psi}_{i l h} & \mathrm{Q}_{i} M_{i l} & \mathrm{Q}_{i} D_{i l} \\
* & * & * & * & -2 I & 0 \\
* & * & * & * & * & -\mathrm{Q}_{i}
\end{array}\right]<0,} \\
& l, a, h=1,2, \ldots, N_{i}, \quad i=1,2, \ldots, m,
\end{aligned}
$$

where

$$
\begin{gathered}
\bar{\Upsilon}_{i a l}=-B_{i a} R B_{i l}^{T}-B_{i l} R B_{i a}^{T}+P_{i}^{-1} A_{i l}^{T}+A_{i l} P_{i}^{-1}+P_{i}^{-1}, \\
\bar{\Psi}_{i l h}=Q_{i}+A_{i l}^{T} Q_{i}-C_{i h}^{T} G_{i l}^{T}+Q_{i} A_{i l}-G_{i l} C_{i h}+N_{i l}^{T} N_{i l}, \\
G_{i l}=Q_{i} L_{i l} .
\end{gathered}
$$

Once one has $P_{i}, Q_{i}$ from (40), then the controllers (14) can be constructed.

Remark 8 . The design procedure can be summarized by the following steps.

Step 1. Design the combined switching controller (14) by choosing the symmetric matrix $R>0$.

Step 2. Check the parameters of the combined switching controller (14) by solving the LMIs in Theorem 7.

Step 3. Design the law of (31) according to Theorem 7.

Remark 9. If (40) holds for some $i$, which is the condition for the observer-based robust control problem of nonswitched system to be solvable, then no switching is needed. Obviously, (40) is much weaker than the condition for nonswitched systems because (40) allows the case that the observer-based robust control problem is solvable for no subsystems.

\section{Illustrative Examples and Results}

In order to demonstrate the efficiency and feasible performance of the proposed control synthesis, now we consider the model as follows:

$$
\begin{aligned}
& R_{1}^{1}: \text { if } z_{2}(t) \text { is } M_{11}^{1}, \\
& \text { then } \\
& \dot{x}(t)= A_{11} x(t)+D_{11} x(t-\tau)+B_{11} u_{11}(t)+\bar{D}_{1} \omega(t), \\
& y(t)= C_{11} x(t) ; \\
& R_{1}^{2}: \text { if } z_{2}(t) \text { is } M_{11}^{2}, \\
& \quad \text { then } \\
& \dot{x}(t)=A_{12} x(t)+D_{12} x(t-\tau)+B_{12} u_{12}(t)+\bar{D}_{1} \omega(t), \\
& y(t)=C_{12} x(t) ;
\end{aligned}
$$




$$
\begin{aligned}
R_{2}^{1}: & \text { if } z_{2}(t) \text { is } M_{21}^{1}, \\
& \text { then } \\
\dot{x}(t)= & A_{21} x(t)+D_{21} x(t-\tau)+B_{21} u_{21}(t)+\bar{D}_{2} \omega(t), \\
y(t)= & C_{21} x(t) ; \\
R_{2}^{2}: & \text { if } z_{2}(t) \text { is } M_{21}^{2}, \\
& \text { then } \\
\dot{x}(t)= & A_{22} x(t)+D_{22} x(t-\tau)+B_{22} u_{22}(t)+\bar{D}_{2} \omega(t), \\
y(t)= & C_{22} x(t) .
\end{aligned}
$$

Also, this model is used for the extra design of the switching law (23) of the state-dependent form. For the purpose of comparing with SF system, Example 1 in [16] can be described for the following.

Region Rule 1. If $z_{2}(t) \geq 1$, then the following is given.

Local Region Rule 1. If $z_{2}(t)$ is $h_{11}\left(x_{2}(t)\right)$,

then

$$
\begin{gathered}
\dot{x}(t)=A_{11} x(t)+D_{11} x(t-\tau)+B_{11} u(t)+\bar{D}_{1} \omega(t), \\
y(t)=C_{11} x(t) .
\end{gathered}
$$

Local Region Rule 2. If $z_{2}(t)$ is $h_{12}\left(x_{2}(t)\right)$,

then

$$
\begin{gathered}
\dot{x}(t)=A_{12} x(t)+D_{12} x(t-\tau)+B_{12} u(t)+\bar{D}_{1} \omega(t), \\
y(t)=C_{12} x(t) .
\end{gathered}
$$

Region Rule 2. If $z_{2}(t)<1$, then the following is given.

Local Region Rule 1. If $z_{2}(t)$ is $h_{21}\left(x_{2}(t)\right)$,

then

$$
\begin{gathered}
\dot{x}(t)=A_{21} x(t)+D_{21} x(t-\tau)+B_{21} u(t)+\bar{D}_{2} \omega(t), \\
y(t)=C_{21} x(t) .
\end{gathered}
$$

Local Region Rule 2. If $z_{2}(t)$ is $h_{22}\left(x_{2}(t)\right)$,

then

$$
\begin{gathered}
\dot{x}(t)=A_{22} x(t)+D_{22} x(t-\tau)+B_{22} u(t)+\bar{D}_{2} \omega(t), \\
y(t)=C_{22} x(t) .
\end{gathered}
$$

Obviously, switching fuzzy system of Example 1 in [16] is switching between each of the sub fuzzy systems and depending on region fuzzy rules.
We have

$$
\begin{gathered}
A_{11}=\left[\begin{array}{cc}
-11.5 & 4 \\
-0.843 & -1.0493
\end{array}\right], \quad B_{11}=\left[\begin{array}{c}
-10.01 \\
-0.4926
\end{array}\right], \\
A_{12}=\left[\begin{array}{cc}
-4.8 & 1 \\
-0.232 & -1.4312
\end{array}\right], \quad B_{12}=\left[\begin{array}{c}
-10.2 \\
-0.1516
\end{array}\right], \\
A_{21}=\left[\begin{array}{cc}
-10 & 0.6 \\
-0.6 & -0.8
\end{array}\right], \quad B_{21}=\left[\begin{array}{c}
-3.4 \\
-1.7
\end{array}\right], \\
A_{22}=\left[\begin{array}{cc}
-12 & 1.2 \\
-2 & -4.529
\end{array}\right], \quad B_{22}=\left[\begin{array}{c}
-1.2 \\
-0.1765
\end{array}\right], \\
D_{11}=\left[\begin{array}{cc}
0.4 & 0.2 \\
0.1 & 0.2
\end{array}\right], \quad D_{12}=\left[\begin{array}{cc}
-0.5 & 0.7 \\
-0.1 & -0.4
\end{array}\right], \\
D_{21}=\left[\begin{array}{cc}
0.2 & 0.3 \\
-0.2 & -0.2
\end{array}\right], \quad D_{22}=\left[\begin{array}{cc}
0.2 & 0.3 \\
0.1 & 0.4
\end{array}\right], \\
C_{11}=\left[\begin{array}{ll}
2 & -1
\end{array}\right], \quad C_{12}=\left[\begin{array}{ll}
-0.8 & 0.1
\end{array}\right], \\
C_{21}=\left[\begin{array}{ll}
5 & -1
\end{array}\right], \quad C_{22}=\left[\begin{array}{ll}
1.6 & 4
\end{array}\right], \\
M_{11}=M_{12}=\left[\begin{array}{cc}
-0.1125 & 1 \\
1 & 0
\end{array}\right], \quad M_{21}=M_{22}=\left[\begin{array}{ll}
0.01 & 1 \\
1 & 0
\end{array}\right], \\
N_{11}=N_{12}=\left[\begin{array}{cc}
1 & 0.2 \\
0 & 0
\end{array}\right], \quad N_{21}=N_{22}=\left[\begin{array}{cc}
0.5 & 1 \\
0 & 0
\end{array}\right], \\
F_{11}(t)=F_{12}(t)=F_{21}(t)=F_{22}(t)=\left[\begin{array}{cc}
\sin (t) & 0 \\
0 & \cos (t)
\end{array}\right], \\
\bar{D}_{1}=\bar{D}_{2}=(1), \quad \quad \theta(t)=1 . \\
\omega_{1}(t)=\omega_{2}(t)=\frac{\cos t^{2},}{\sin t},
\end{gathered}
$$

The fuzzy sets of " $M_{11}^{1}, M_{11}^{2}, M_{21}^{1}, M_{21}^{2}$ ” are represented by the following membership functions, respectively:

$$
\begin{gathered}
M_{11}^{1}\left(z_{2}(t)\right)=h_{11}\left(z_{2}(t)\right)=1-\frac{1}{1+e^{-2 z_{2}(t)}}, \\
M_{11}^{2}\left(z_{2}(t)\right)=h_{12}\left(z_{2}(t)\right)=\frac{1}{1+e^{-2 z_{2}(t)}}, \\
M_{21}^{1}\left(z_{2}(t)\right)=h_{21}\left(z_{2}(t)\right)=1-\frac{1}{1+e^{\left(-2\left(z_{2}(t)-0.3\right)\right)}}, \\
M_{21}^{2}\left(z_{2}(t)\right)=h_{22}\left(z_{2}(t)\right)=\frac{1}{1+e^{\left(-2\left(z_{2}(t)-0.3\right)\right)}},
\end{gathered}
$$

Consider the closed-loop systems (13). Choosing $l, a, h=$ $1,2 \ldots, N_{i}, i=1,2, R=8.4317$, and $\tau=1$ for (40), we can have

$$
\begin{gathered}
P_{1}=\left[\begin{array}{ll}
524.5761 & 342.5681 \\
342.5681 & 578.1421
\end{array}\right], \\
P_{2}=\left[\begin{array}{ll}
631.1245 & 412.7413 \\
412.7413 & 514.2356
\end{array}\right], \\
Q_{1}=\left[\begin{array}{ll}
5.3152 & 0.2942 \\
0.2942 & 2.0512
\end{array}\right], \quad Q_{2}=\left[\begin{array}{ll}
4.8514 & 0.3571 \\
0.3571 & 3.6316
\end{array}\right] .
\end{gathered}
$$




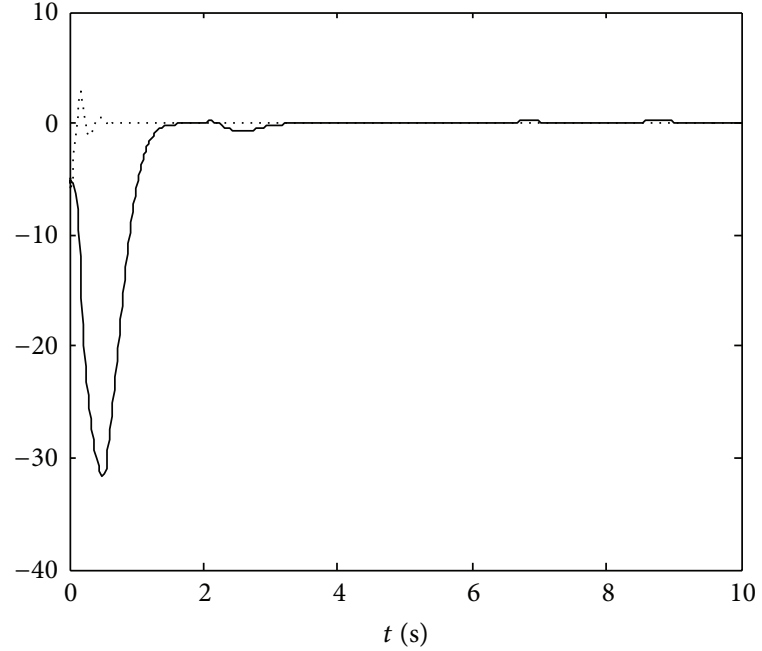

(a)

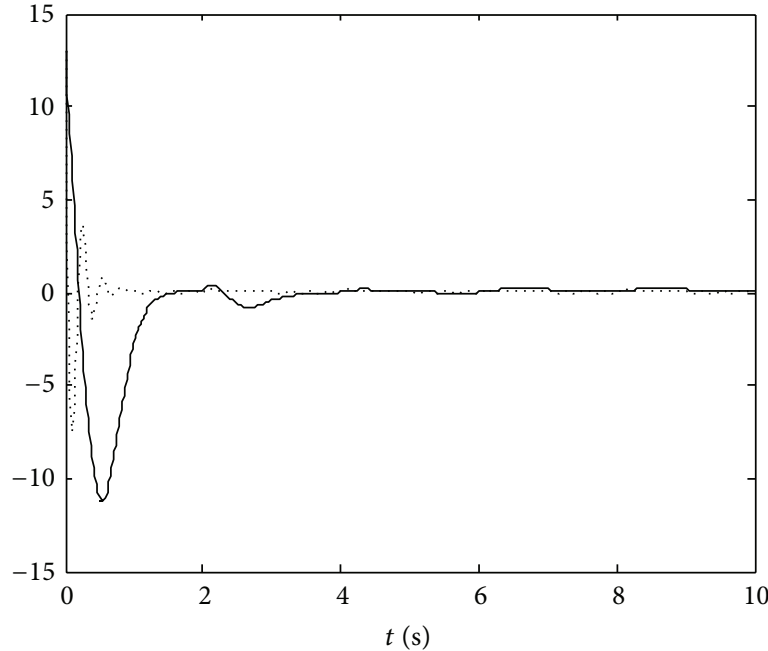

(b)

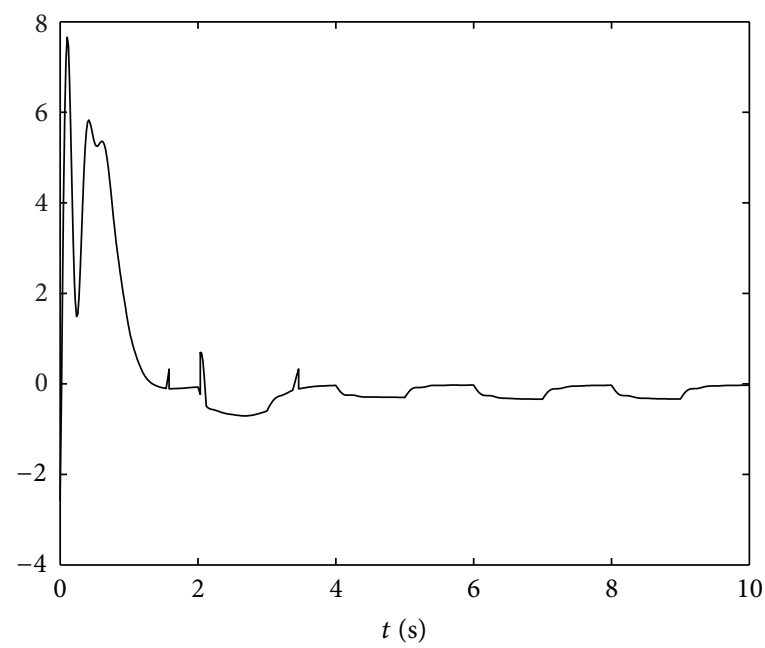

(c)

Figure 2: (a) System state $x_{1}(t)$ (solid line) and observer state $\widehat{x}_{1}(t)$ (dotted line) with the combined switching controller. (b) System state $x_{2}(t)$ (solid line) and observer state $\widehat{x}_{2}(t)$ (dotted line) with the combined switching controller. (c) Control signal of the combined switching controller $u_{i l}(t)$.

And we have the candidate observer gains via arbitrary switching as

$$
\begin{array}{ll}
L_{11}=\left[\begin{array}{l}
0.8921 \\
0.2324
\end{array}\right], & L_{12}=\left[\begin{array}{l}
5.0821 \\
0.5973
\end{array}\right], \\
L_{21}=\left[\begin{array}{l}
0.9075 \\
0.3032
\end{array}\right], & L_{22}=\left[\begin{array}{l}
0.8132 \\
0.2019
\end{array}\right] .
\end{array}
$$

Taking the initial condition as $x(0)=[-5,13]^{T}$, then the simulation result for the combined switching controller (14) of SF time-delay system (5) is depicted in Figure 2.

To investigate the effectiveness of the proposed SF system here, we now compare the switching fuzzy system in [16-19]. The SF model is used for the extra design of the switching law (23), but switching fuzzy system switchs depending on region fuzzy rules. For the same data of Example 1 in [16] and the same initial condition $x(0)=[-5,13]^{T}$, the simulation result is depicted in Figure 3. It can be seen that the proposed SF system provides the best performance in terms of faster transient responses and shorter settling time.

The traditional PDC fuzzy controller is employed to handle the same SF time-delay system in [34]. According to [34], the global control of the traditional PDC fuzzy controller is

$$
u(t)=\sum_{i=1}^{N_{i}} \sum_{l=1}^{N_{i}} v_{i} h_{i l} K_{i l} \widehat{x}(t)
$$

where

$$
v_{j}(z(t))= \begin{cases}1, & z(t) \in \text { Region } j \\ 0, & \text { otherwise. }\end{cases}
$$




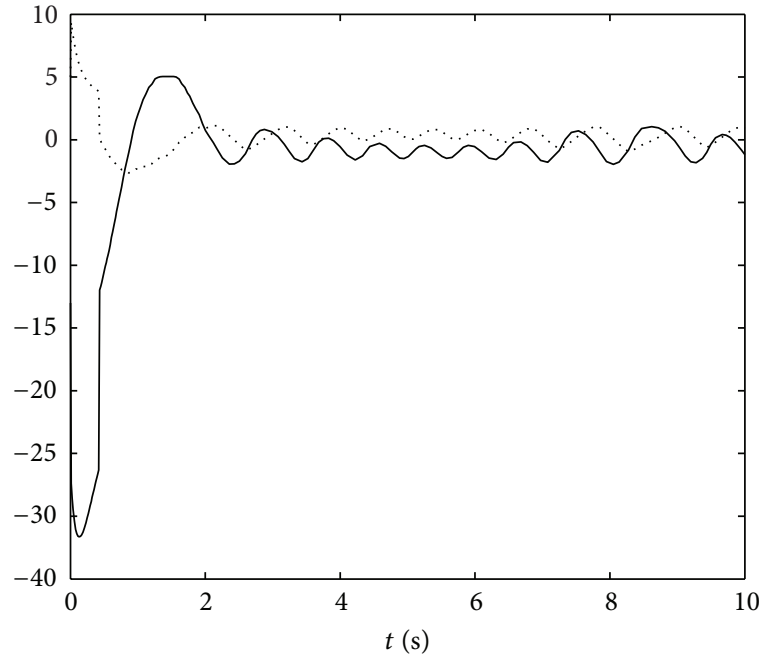

(a)

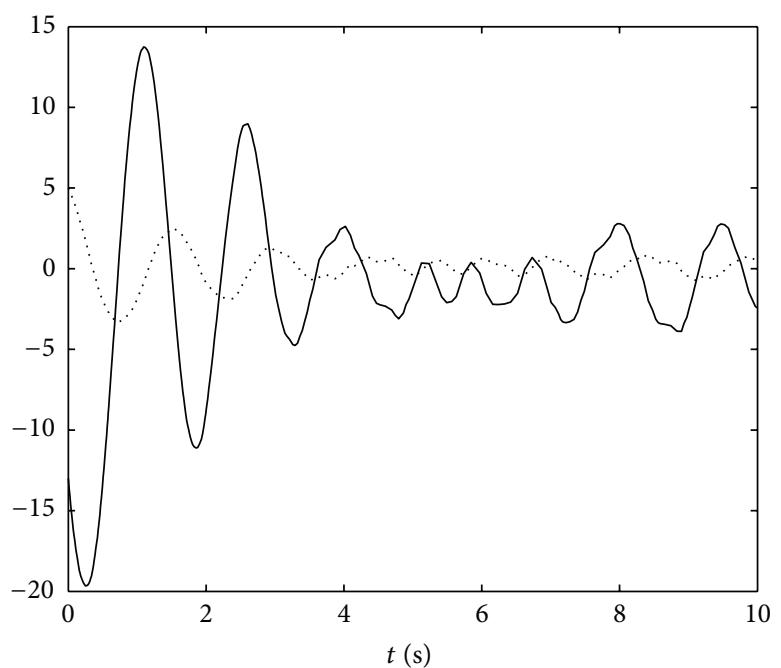

(b)

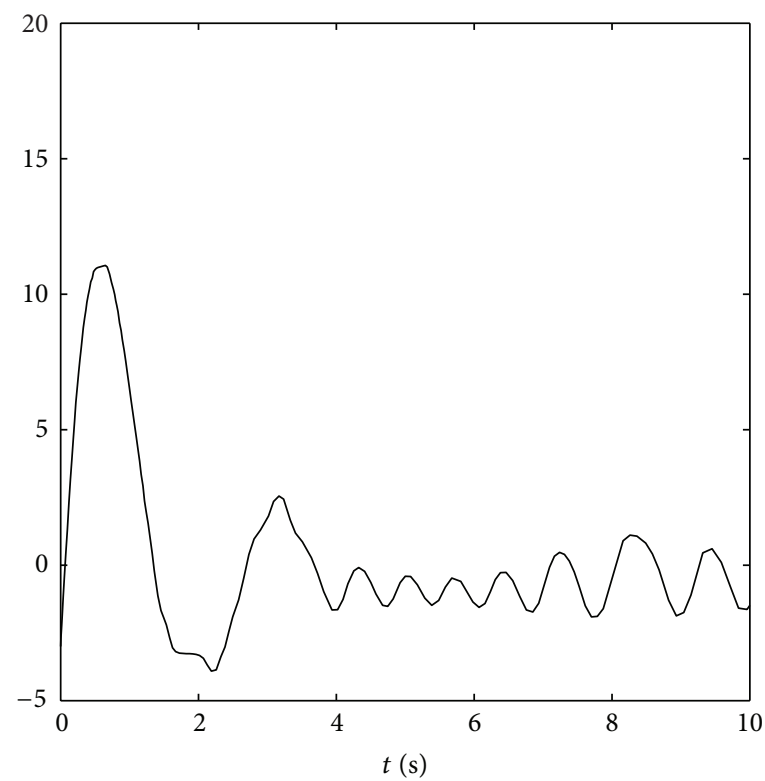

(c)

Figure 3: (a) System state $x_{1}(t)$ (solid line) and observer state $\widehat{x}_{1}(t)$ (dotted line) of switching fuzzy system with its switching controller in [16]. (b) System state $x_{2}(t)$ (solid line) and observer state $\widehat{x}_{2}(t)$ (dotted line) of switching fuzzy system with its switching controller in [16]. (c) Control signal of the switching controller $u(t)$ in [16] of switching fuzzy system.

The state feedback gains of subsystems are obtained as follows:

$$
\begin{aligned}
& K_{11}=\left[\begin{array}{ll}
-0.542 & -0.125
\end{array}\right], \\
& K_{12}=\left[\begin{array}{ll}
-0.6532 & -7.4367
\end{array}\right] \\
& K_{21}=\left[\begin{array}{ll}
-3.4 & -5.32
\end{array}\right], \\
& K_{22}=\left[\begin{array}{ll}
-3.12 & -4.21
\end{array}\right] .
\end{aligned}
$$

Figure 4 shows the SF system responses and the control signals under the same initial condition $x(0)=[-5,13]^{T}$. Referring to Figure 2, it can be seen that the performance of the combined switching controller (14) is slightly better in terms of transient response time.

\section{Conclusion}

In this paper, the observer-based robust control for SF system with time delay is investigated. We used the multiple Lyapunov function technique and a switching law to design a control law such that the system along with fuzzy observer is asymptotically stable. The combined switching controller design problem can be solved efficiently by using linear matrix inequalities and convex optimization techniques. 


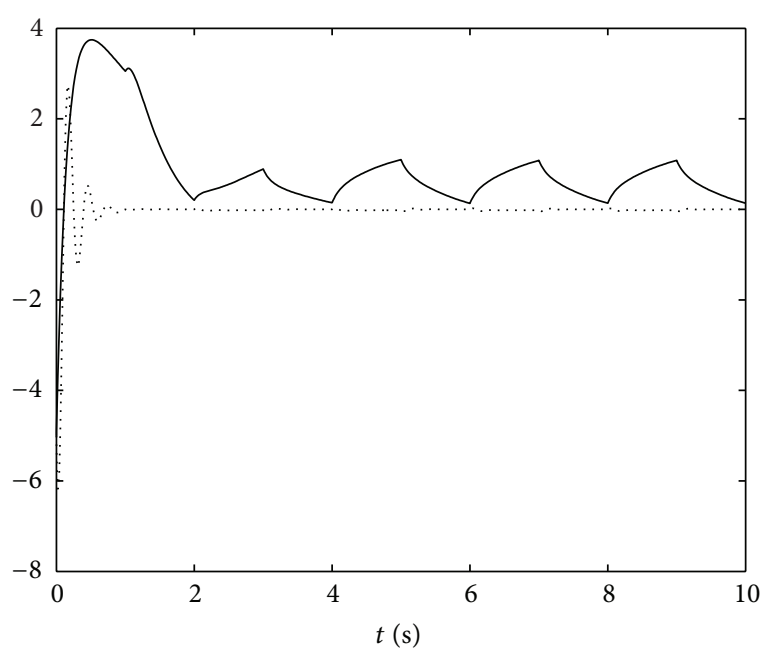

(a)

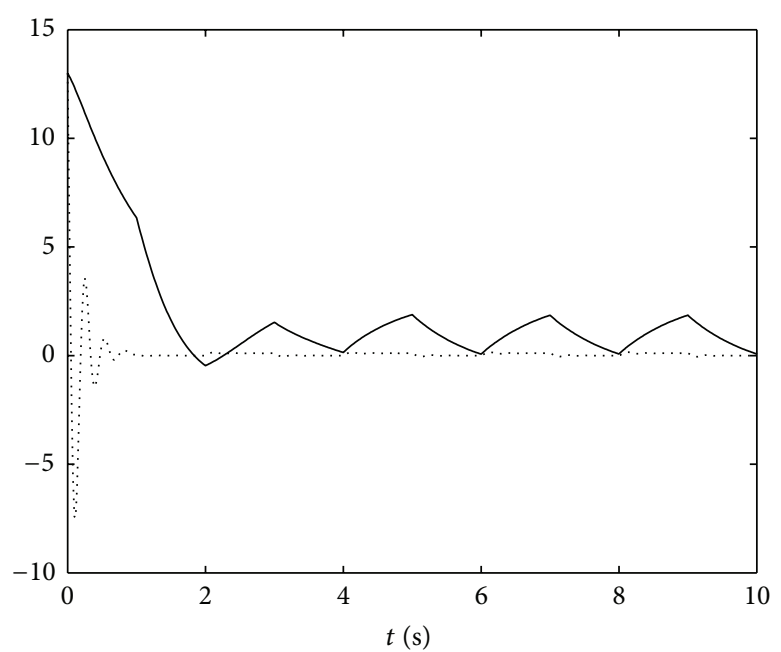

(b)

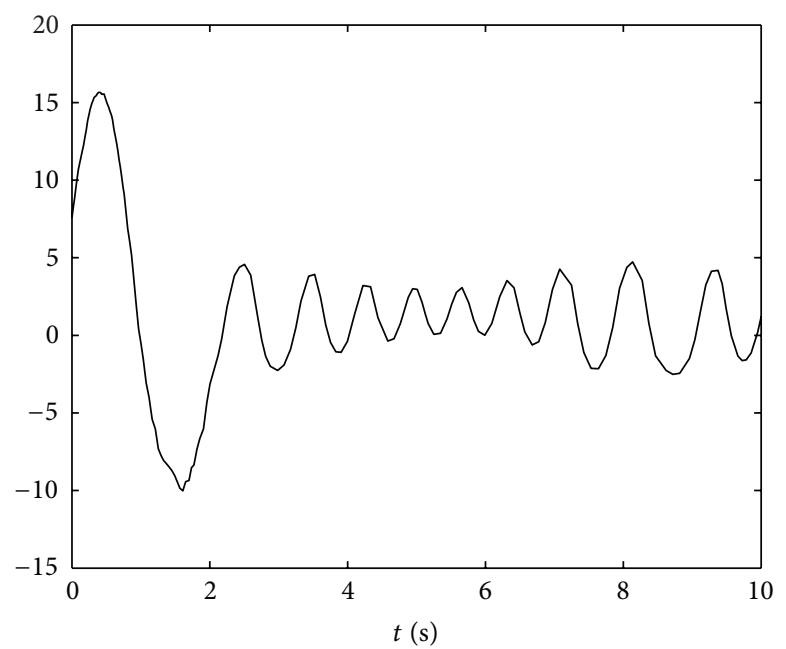

(c)

FIgURE 4: (a) System state $x_{1}(t)$ (solid line) and observer state $\widehat{x}_{1}(t)$ (dotted line) with the traditional PDC fuzzy controller of SF system. (b) System state $x_{2}(t)$ (solid line) and observer state $\widehat{x}_{2}(t)$ (dotted line) with the traditional PDC fuzzy controller of SF system. (c) Control signal of the traditional PDC fuzzy controller $u(t)$ of SF system.

The simulation example shows the validity of the switching control law.

\section{Acknowledgments}

This paper is supported by the National Natural Science Foundation under Grant no. 61004039, the Science Foundation of the Educational Department of Liaoning Province under Grant no. 2010375, and the Excellent Talented Person Foundation of the Educational Department of Liaoning Province under Grant no. LJQ2011127. The authors also gratefully acknowledge the helpful comments and suggestions of the reviewers, which have improved the presentation.

\section{References}

[1] X.-M. Sun, G.-P. Liu, W. Wang, and D. Rees, "L2-gain of systems with input delays and controller temporary failure: zero-order hold model," IEEE Transactions on Control Systems Technology, vol. 19, no. 3, pp. 699-706, 2011.

[2] L. Wu and W. X. Zheng, "Weighted $H_{\infty}$ model reduction for linear switched systems with time-varying delay," Automatica, vol. 45, no. 1, pp. 186-193, 2009.

[3] L. Wu, Z. Feng, and W. X. Zheng, "Exponential stability analysis for delayed neural networks with switching parameters: average dwell time approach," IEEE Transactions on Neural Networks, vol. 21, no. 9, pp. 1396-1407, 2010.

[4] M. S. Ali, "On exponential stability of neutral delay differential system with nonlinear uncertainties," Communications in Nonlinear Science and Numerical Simulation, vol. 17, no. 6, pp. 25952601, 2012.

[5] M. Liu, D. W. C. Ho, and Y. Niu, "Stabilization of Markovian jump linear system over networks with random communication delay," Automatica, vol. 45, no. 2, pp. 416-421, 2009.

[6] M. Liu, P. Shi, L. Zhang, and X. Zhao, "Fault-tolerant control for nonlinear Markovian jump systems via proportional and 
derivative sliding mode observer technique," IEEE Transactions on Circuits and Systems. I, vol. 58, no. 11, pp. 2755-2764, 2011.

[7] J. Zhao and G. M. Dimirovski, "Quadratic stability of a class of switched nonlinear systems," IEEE Transactions on Automatic Control, vol. 49, no. 4, pp. 574-578, 2004.

[8] L. G. Wu, Wei Xing Zheng, and H. J. Gao, "Dissipativity-based sliding mode control of switched stochastic systems," IEEE Transactions on Automatic Control, vol. 58, no. 3, pp. 785-791, 2013.

[9] J. Liu, X. Liu, and W.-C. Xie, "Input-to-state stability of impulsive and switching hybrid systems with time-delay," Automatica, vol. 47, no. 5, pp. 899-908, 2011.

[10] D. Xie and X. Chen, "Observer-based switched control design for switched linear systems with time delay in detection of switching signal," IET Control Theory \& Applications, vol. 2, no. 5, pp. 437-445, 2008.

[11] X.-M. Sun, G.-P. Liu, D. Rees, and W. Wang, "Stability of systems with controller failure and time-varying delay," IEEE Transactions on Automatic Control, vol. 53, no. 10, pp. 23912396, 2008.

[12] X.-M. Sun and W. Wang, "Integral input-to-state stability for hybrid delayed systems with unstable continuous dynamics," Automatica, vol. 48, no. 9, pp. 2359-2364, 2012.

[13] Q.-K. Li, J. Zhao, and G. M. Dimirovski, "Robust tracking control for switched linear systems with time-varying delays," IET Control Theory \& Applications, vol. 2, no. 6, pp. 449-457, 2008.

[14] H. K. Lam and F. H. F. Leung, "Fuzzy rule-based combination of linear and switching state-feedback controllers," Fuzzy Sets and Systems, vol. 156, no. 2, pp. 153-184, 2005.

[15] L. Wu, X. Su, P. Shi, and J. Qiu, "A new approach to stability analysis and stabilization of discrete-time T-S fuzzy timevarying delay systems," IEEE Transactions on Systems, Man, and Cybernetics B, vol. 41, no. 1, pp. 273-286, 2011.

[16] K. Tanaka, M. Iwasaki, and H. O. Wang, "Switching control of an R/C hovercraft: stabilization and smooth switching," IEEE Transactions on Systems, Man, and Cybernetics B, vol. 31, no. 6, pp. 853-863, 2001.

[17] K. Tanaka, M. Iwasaki, and H. O. Wang, "Stability and smoothness conditions for switching fuzzy systems," in Proceedings of the American Control Conference, pp. 2474-2478, June 2000.

[18] W.-J. Wang, Y.-J. Chen, and C.-H. Sun, "Relaxed stabilization criteria for discrete-time T-S fuzzy control systems based on a switching fuzzy model and piecewise Lyapunov function," IEEE Transactions on Systems, Man, and Cybernetics B, vol. 37, no. 3, pp. 551-559, 2007.

[19] D. J. Choi and P. Park, "Guaranteed cost controller design for discrete-time switching fuzzy systems," IEEE Transactions on Systems, Man, and Cybernetics B, vol. 34, no. 1, pp. 110-119, 2004.

[20] X. J. Su, P. Shi, L. G. Wu, and Y. D. Song, "A novel approach to filter design for T-S fuzzy discrete-time systems with timevarying delay," IEEE Transactions on Fuzzy Systems, vol. 20, no. 6, pp. 1114-1129, 2012.

[21] L. Wu and D. W. C. Ho, "Fuzzy filter design for Itô stochastic systems with application to sensor fault detection," IEEE Transactions on Fuzzy Systems, vol. 17, no. 1, pp. 233-242, 2009.

[22] L. Wu and W. X. Zheng, "L2-Lo control of nonlinear fuzzy itô stochastic delay systems via dynamic output feedback," IEEE Transactions on Systems, Man, and Cybernetics B, vol. 39, no. 5, pp. 1308-1315, 2009.
[23] T.-C. Lin, S.-W. Chang, and C.-H. Hsu, "Robust adaptive fuzzy sliding mode control for a class of uncertain discretetime nonlinear systems," International Journal of Innovative Computing, Information and Control, vol. 8, no. 1 A, pp. 347359, 2012.

[24] W.-H. Ho, S.-H. Chen, I.-T. Chen, J.-H. Chou, and C.-C. Shu, "Design of stable and quadratic-optimal static output feedback controllers for TS-fuzzy-model-based control systems: an integrative computational approach," International Journal of Innovative Computing, Information and Control, vol. 8, no. $1 \mathrm{~A}$, pp. 403-418, 2012.

[25] X. Su, L. Wu, and P. Shi, "Senor networks with random link failures: distributed filtering for T-S fuzzy systems," IEEE Transactions on Industrial Informatics, 2012.

[26] X. Su, L. Wu, P. Shi, and Y. D. Song, " $H_{\infty}$ model reduction of TS fuzzy stochastic systems," IEEE Transactions on Systems, Man, and Cybernetics B, vol. 42, no. 6, pp. 1574-1585, 2012.

[27] H. Yang, X. Sun, and X. Liu, "Stabilization and switched control for a class of switched fuzzy systems," International Journal of Innovative Computing, Information and Control, vol. 5, no. 10, pp. 3099-3108, 2009.

[28] J. Lian, Z. Feng, and P. Shi, "Observer design for switched recurrent neural networks: an average dwell time approach," IEEE Transactions on Neural Networks, vol. 22, no. 10, pp. 15471556, 2011.

[29] D. Xie and X. Chen, "Observer-based switched control design for switched linear systems with time delay in detection of switching signal," IET Control Theory \& Applications, vol. 2, no. 5, pp. 437-445, 2008.

[30] A. Kruszewski, W. J. Jiang, E. M. Fridman, J. P. P. Richard, and A. K. A. Toguyéni, "A switched system approach to exponential stabilization through communication network," IEEE Transactions on Control Systems Technology, vol. 20, no. 4, pp. 887-900, 2012.

[31] C. Lin, Q.-G. Wang, T. H. Lee, Y. He, and B. Chen, “Observerbased $H_{\infty}$ fuzzy control design for T-S fuzzy systems with state delays," Automatica, vol. 44, no. 3, pp. 868-874, 2008.

[32] S. Tong and Y. Li, "Observer-based fuzzy adaptive robust control of nonlinear systems with time delays and unmodeled dynamics," Neurocomputing, vol. 74, no. 1-3, pp. 369-378, 2010.

[33] L. Wu, X. Su, P. Shi, and J. Qiu, "Model approximation for discrete-time state-delay systems in the TS fuzzy framework," IEEE Transactions on Fuzzy Systems, vol. 19, no. 2, pp. 366-378, 2011.

[34] Y. Liu and J. Zhao, "Observer-based feedback control for switching fuzzy time-delay systems," Control Theory and Applications, vol. 26, no. 3, pp. 274-276, 2009. 


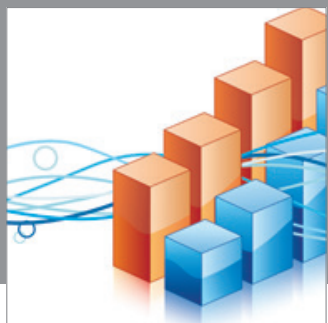

Advances in

Operations Research

mansans

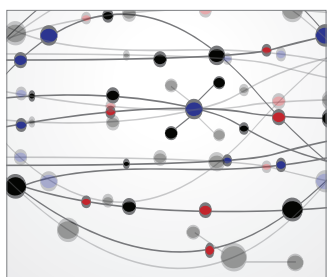

The Scientific World Journal
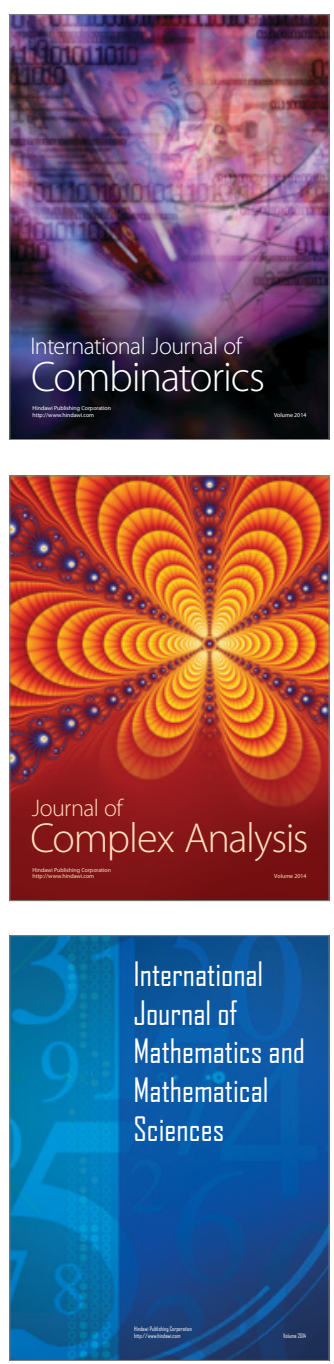
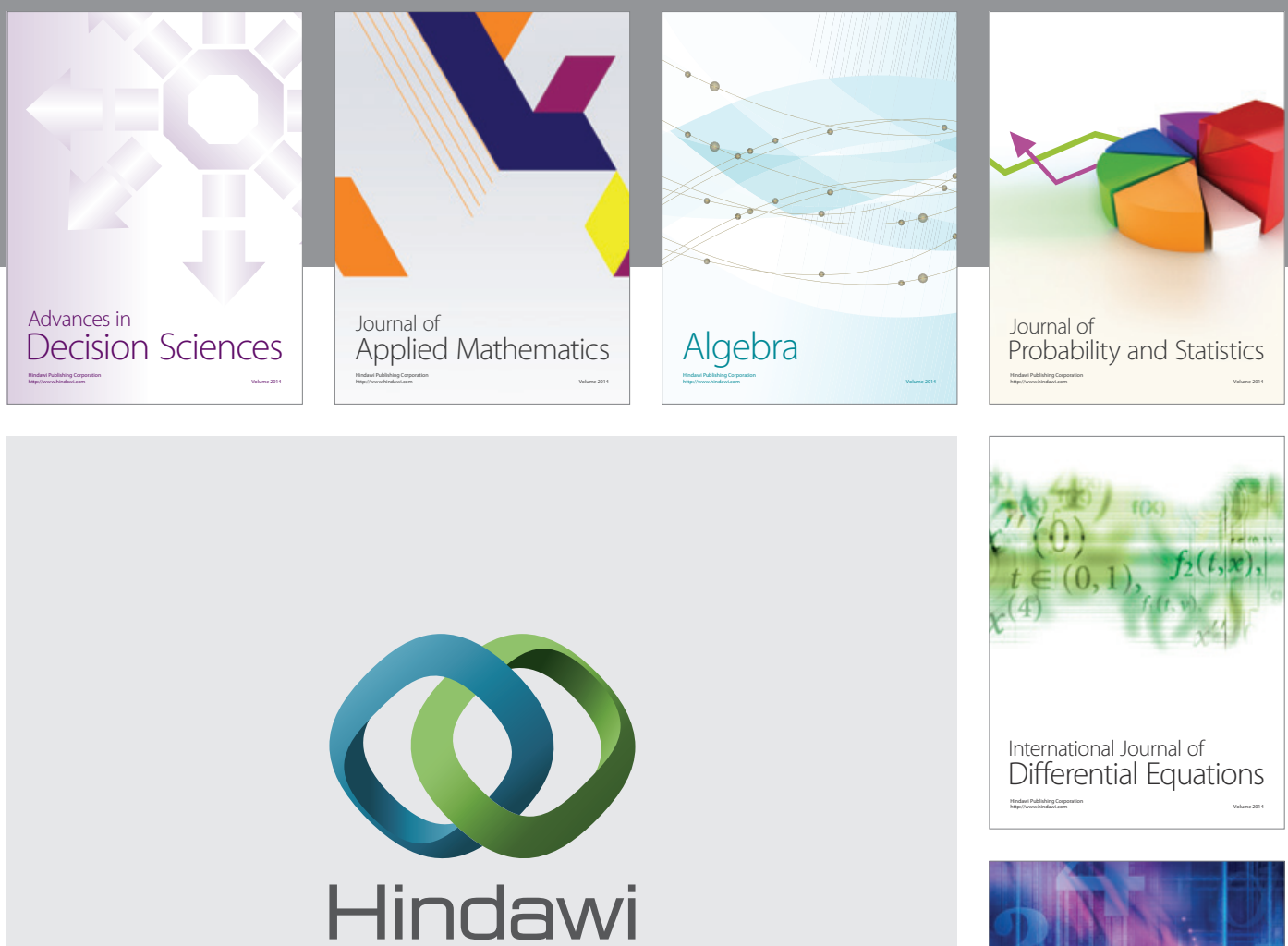

Submit your manuscripts at http://www.hindawi.com
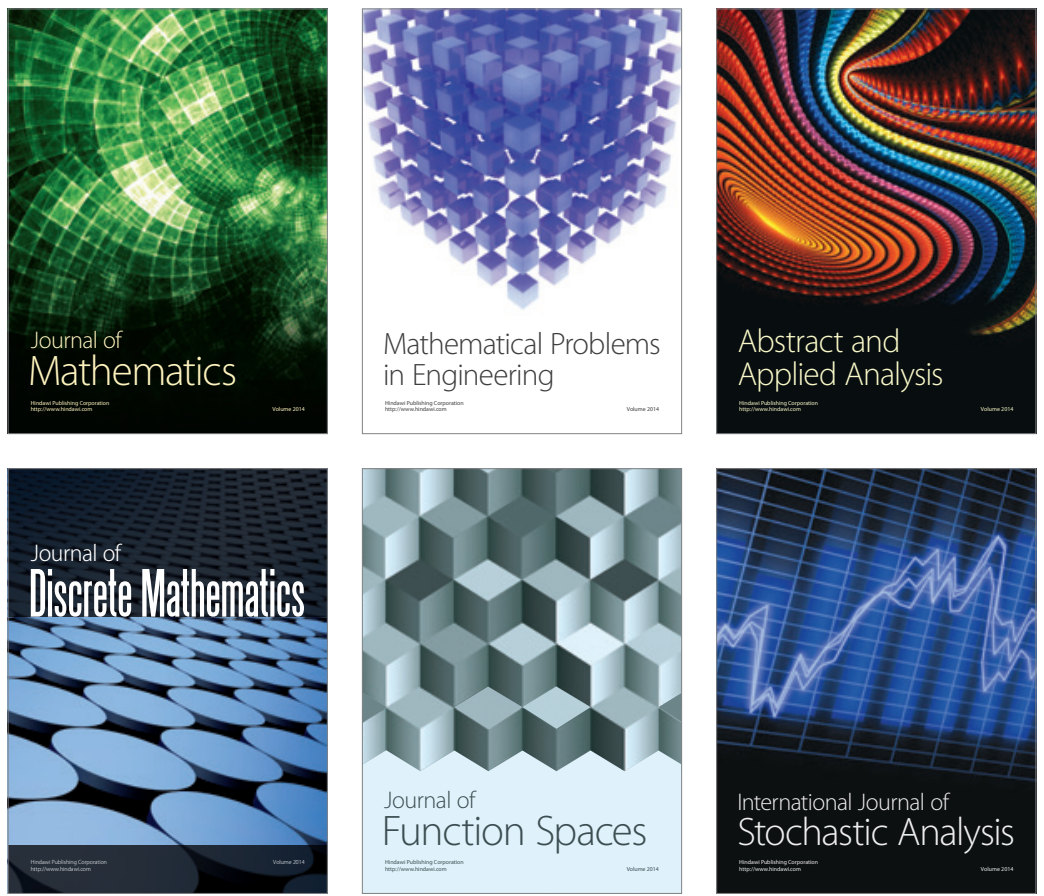

Journal of

Function Spaces

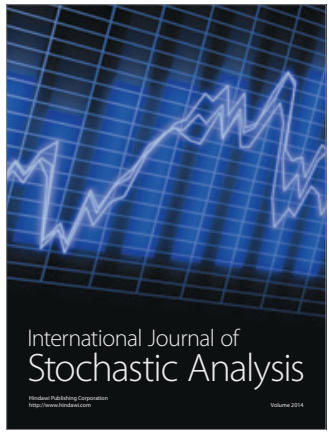

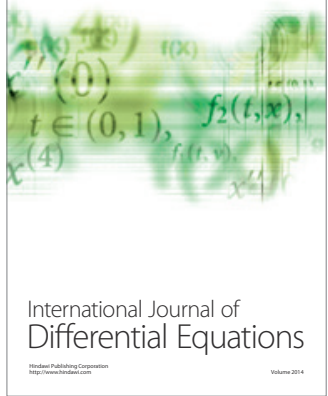
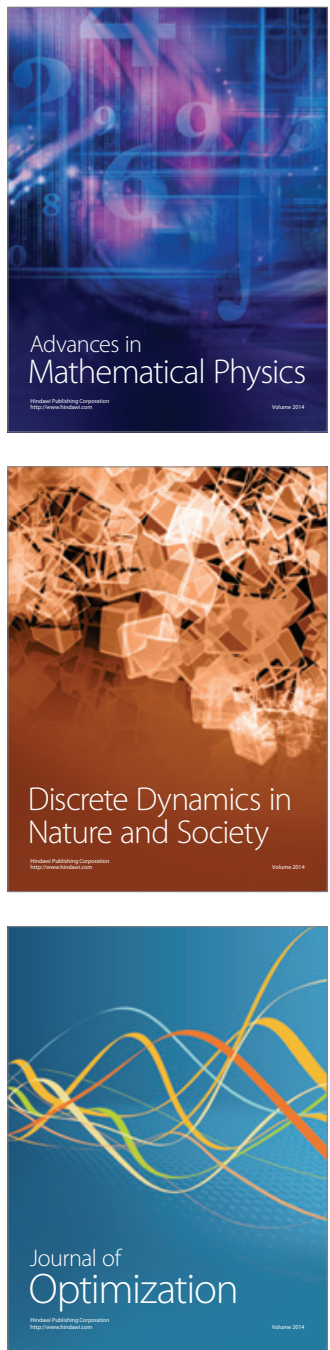